\title{
Design and Implementation of an Automatic Test Paper Generation System Based on ant colony optimization
}

\author{
Yingjie Fu \\ Department of Teaching Affairs, Jilin Agricultural University, Changchun, China \\ jlaufyj@126.com
}

Keywords: automatic test paper generation; ant colony optimization; item bank; data structure

\begin{abstract}
The paper proposed a model of automatic test paper generation system since the traditional test was time-consuming, labor-intensive and unstable. Based on the demand of the automatic test paper generation system the model explored the setting of the initial value information of the ant colony optimization and updated the rules so that the test results could he feed backed to the system. This model not only effectively solved the problem of the auto-generating test-paper but also improved the autonomous learning ability of the system, which was more intelligent to improve the performance. The practical test proves that the system has achieved the expected goal with high quality and high efficiency. The practical test shows that the system has advantages like fast generation of test papers, low repetition of the selected papers and effective colony algorithm. Examinations designed based on this system an achieve the expected testing goals
\end{abstract}

\section{Introduction}

With the rapid development of computer technology and database technology, the people have been occurred great changes in various fields of life, especially in the field of education. The computer-aided education has become one of the most important tools in the common day-to-day work of the major schools. The massive rise in the number of students increased teaching load at the same time and the direct result of the various institutions of higher learning takes a lot of human, financial and material resources in the organization of the examination affairs. Therefore, this paper researched and designed the automatic test paper system which based on computer information technology and could improve the efficient direction of development of the examination affairs, in institutions of higher learning in the teaching process, because a good test paper generation system can accurately test teachers teaching results and student achievement. To develop a test paper system which can evaluate the teaching levels objectively is an important subject for the teaching reform. Purpose of this paper is to study and develop a set of constraints to the conditions setoff the user automatically and quickly generate a set of high-quality papers, to check the students test the true purpose of learning ability. Automatic generation of efficiency and quality depends largely on the Question Bank questions arithmetic in the design and selection, so the main contents of this paper is the mathematical model and the test paper questions arithmetic.

\section{System Model Design}

\section{A. Requirement Analysis}

Automatic test paper generation system shall establish question banks for various subjects based on the actual need of the school, through which, teachers of different subjects can establish a test bank for their own course bank complying with their requirements. The contents, answers and other factors to be considered shall be put in the question bank in the form of fields. An appropriate algorithm of generating test paper shall be designed in order to ensure effective selection of relevant questions in the question bank and generate satisfactory test papers. Finally, a statistical analysis of the grades of students will be completed by the system[1]. User permission shall be set to ensure security of the entire system and the test papers. 


\section{B. Introduction of the System Modules}

The whole system includes item bank management, test paper generation management, statistical analysis of results and system management and other functional modules. The structure diagram is as follows:

Item bank management: management of the item bank is a precondition to normal test paper generation. This module enables professional teachers to create the question bank of their own subject and add, modify, delete, inquire and browse questions in the question bank they established.

Test paper generation management: test paper generation is the nature and core of the system. Users can enter requirements of test paper generation through this module and automatically generate a test paper meeting user's needs by the system

Results statistical analysis: after the students finish the test, teachers can enter their marks into the system by this module. The system will conduct statistical analysis after receiving the mark of each subject and print the analysis results in files. The analysis results can be provided for relevant teacher for further analysis and improvement of the question bank by adjusting the questions based on the analysis results[2].

System management: this module is set to ensure security of the whole system and the test paper. System administrator can add or delete user permission of related operator by this module.

\section{Mathematical Model of Automatic Test Paper Generation System}

Automatic test paper generation refers to the process of choosing a certain amount of test questions in the item bank to form a test paper by the computer. The test paper can both meet examination requirements and users needs[3]. Assume that a test paper contains $m$ questions, and each of which has $n$ attribute indexes, then a test paper is a $m \times n$ matrix, that is:

$$
\mathrm{S}=\left[\begin{array}{cccc}
a_{11} & a_{12} & \cdots & a_{1 n} \\
a_{21} & a_{22} & \cdots & a_{2 n} \\
\cdots & \cdots & \cdots & \cdots \\
a_{m 1} & a_{m 2} & \cdots & a_{m n}
\end{array}\right]
$$

The target matrix shall meet following constraint conditions:

1) Constraint on test score: $K=\sum_{i=1}^{m} a_{i 2}$, the specific value is set by the user.

2) Constraint on types of test questions and score: $Q$ represents the score of the $t$ question, $t$ represents the number of question type, $C$ represents choice question, true or false questions, gap filing question, short-answer question and calculation question. The specific type of questions and the score proportion of all questions are set by the user, then:

$$
\begin{aligned}
& Q_{i}=\sum_{i=1}^{m} C_{i j} \times a_{i 2} \\
& \mathrm{C}_{i j}= \begin{cases}1 & \left(a_{i j}=t\right) \\
0 & \left(a_{i j} \neq t\right)\end{cases}
\end{aligned}
$$

3) Total time: assume that the total time of the test is T0, the required time to complete $X$ test paper is $T(X)$, checkout time is $\beta T(X)$, then the time spent on completion of test paper $X$ is: $\mathrm{T}^{0}(\mathrm{X})=(1+\beta) \mathrm{T}(\mathrm{X})=(1+\beta) \sum_{i=1}^{n} t_{i}(x)$

4) $\mathrm{PS}^{t}$ is used to represent item difficulty distribution, the item difficulty can be defined as: $\mathrm{PS}^{t}=\sum_{i=1}^{m} C_{i j} \times a_{i 2}$; 
If a ${ }^{i 3}$ falls into the range of $i$ grade of difficulty, then $C_{1 i}$ equals to1 or equals to 0 .

5) Restraint on score of knowledge point: assume the sets of key points and secondary key points are $\mathrm{I}^{1}$ and $\mathrm{I}^{2}$, and the corresponding score ranges are $\left[\mathrm{L}^{1}, \mathrm{U}^{1}\right]$ and $\left[\mathrm{L}^{2}, \mathrm{U}^{2}\right]$, then :

$$
\left\{\begin{array}{l}
L_{1} \leq \sum C_{2 i} \times a_{i 2} \leq U_{1} \\
L_{2} \leq \sum C_{3 i} \times a_{i 2} \leq U_{2}
\end{array}\right.
$$

6) Restraint on knowledge coverage: it is represented by symbol R, whose definition is as follows:
$\mathrm{R}$
$=$ number of knowledge po int s in the test questions number of all knowledge po ints
$\geq$
r

Under normal circumstances, $r$ is greater than $80 \%$ to ensure coverage of knowledge points of the test paper.

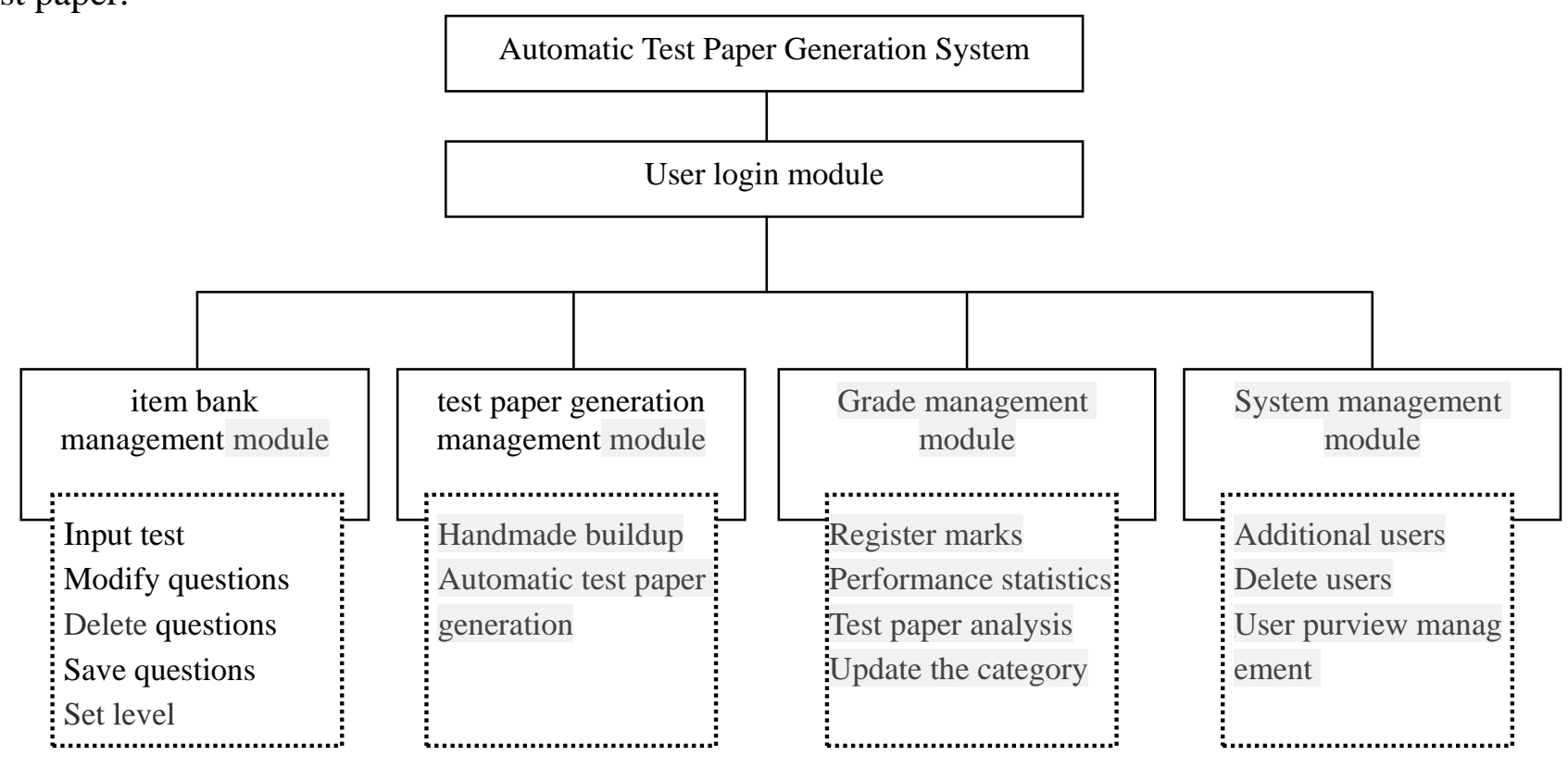

Figure 1. Functional Module Chart of Automatic Test Paper Generation System

\section{Simulation Experiment}

\section{A. Experiment Parameter}

In the improved ant colony algorithm, setting of each parameter can affect the performance of the system. Taking various factors and practices into consideration, the parameters values are as shown in Table I .

TABLE I. INSTRUCTION ON USE OF PARAMETERS

\begin{tabular}{llllll}
\hline $\begin{array}{l}\text { Parameter } \\
\text { name }\end{array}$ & L & $\mathbf{n}$ & $\mathbf{m}$ & $\boldsymbol{\alpha}$ & $\boldsymbol{\beta}$ \\
\hline Value & 10000 & 10 & 2 & 1 & 2 \\
\hline
\end{tabular}

\section{B. Experimental Analysis}

The system uses C \# + SQL Server implementation and the test environment is provided with serve and testing machine environment as shown in Table II. The LoadRunner is used for pressure test, and the test results are shown in Figure 2[4]. 


\begin{tabular}{ll}
\hline & Operating system: Windows Server 2008 \\
Server & CPU: Intel(R) Core(TM) i5-2300 \\
& RAM: 8G \\
& Hard disk: 1T \\
& Database: SQL Server 2008 \\
& Web Server:IIS 7.0 \\
& Operating system: Windows Server 2003 \\
& CPU: Intel(R) Celeron(R) G1101 \\
& RAM: 4G \\
Tester & Hard disk: 80G \\
& LoadRunner: 8.0 \\
& Browser: IE 6.0
\end{tabular}

CPU share (\%)

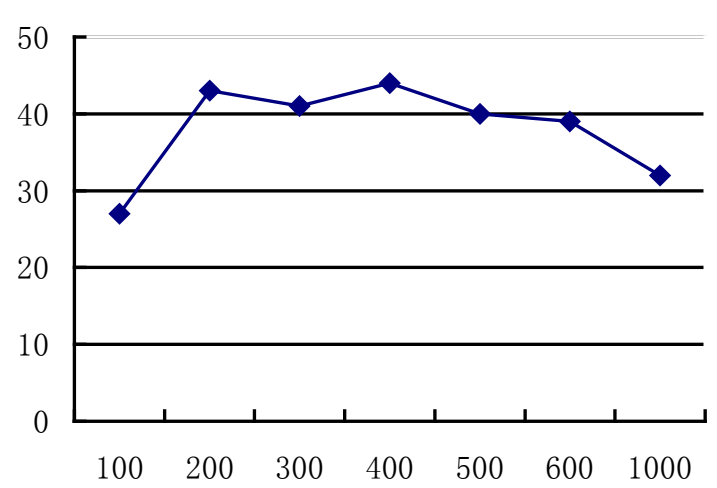

(a) CPU share

Memory stinginess (GB)

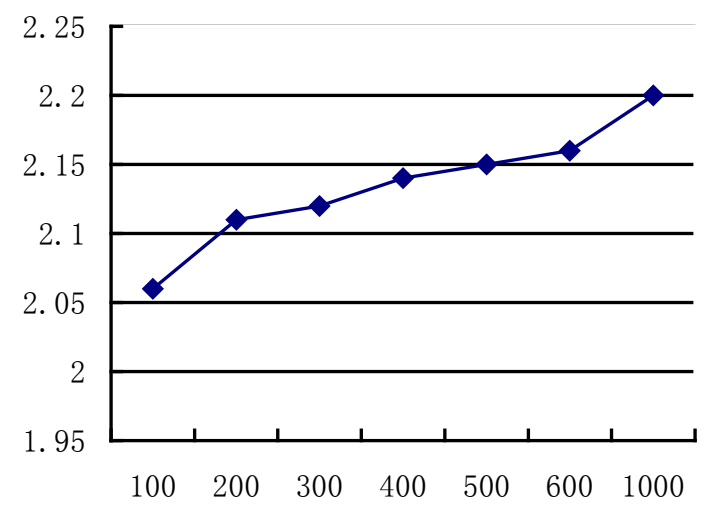

(b) Memory stinginess 


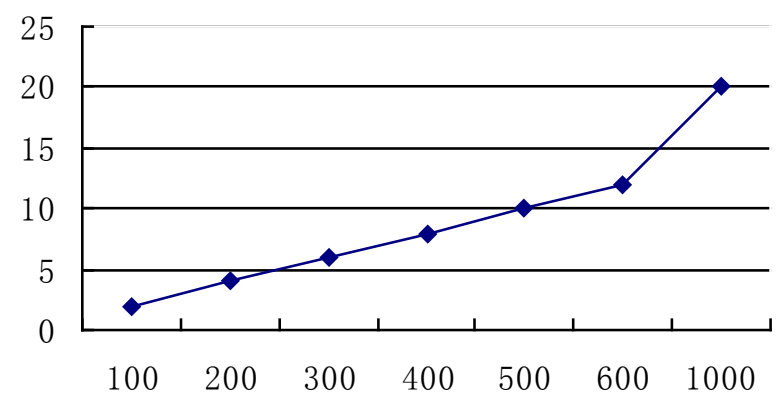

(c) Average save time

Figure 2. Pressure test data analysis

The LoadRunner is used for pressure test under the simulated circumstance where 100,200, 1000 examinees logging into the system, taking the test and submitting test papers at the same time. The reaction and thinking time of users is set at 1 and a set of test paper containing 96 questions and $2844 \mathrm{~KB}$ picture attachment is used for the test. It can be seen that the system successfully completed multi-user examinees log into the system, take the test and save the paper and the database connections are within the upper limit without any server crash and loss of user's paper. Test paper generation is provided with paper requirements and not a single question is chosen continuously in the automatic test paper, which solves the problem of high repetition rate of test questions ${ }^{[5]}$.

\section{Conclusion}

The core of auto-forming test paper system is high-quality item bank and high-efficient algorithm of generating test paper. This paper updates the strategy on the basis of existing ant colony algorithm design and solves the problem of high repetition rate of questions in test paper. With the use of ant colony algorithm, the feedback of examination results to the item bank will be completed, so that the item bank information can truly reflect the test questions information. The experiment shows that, the system can meet the test requirements and achieve test objectives through continuous learning and accumulation.

\section{References}

[1] R. J. Wu, "Study on Intelligently Composing Test Paper Based on Based on Ant Colony Optimization,”Computer Simulation, vol. 28, Aug. 2011, pp. 380-384.

[2] K. Zhang, "Design and Implementation of an Automatic Test Paper Generation System Based on Genetic Algorithms," Computer Engineering \& Science, vol. 34, May 2012, pp. 178-183.

[3] W. H. Qi, “Intelligent Test System Model Based on Ant Colony Optimization,”Application Research of Computers, vol. 30, Mar. 2013, pp. 775-778.

[4] Y. M. Wang, "Research on Intelligent Grouping System Based on Genetic Algorithm,” Journal of Yunnan Nationalities University, vol. 18, Apr. 2009, pp. 173-176.

[5] S. W. Tang, "Study on Intelligent Test Paper Generation Algorithm Based on Ant Colony Optimization,” Computer Era, vol. 10, Oct. 2011, pp.37-39. 